\title{
Green's Function for Dirac Particle in a Non-Abelian SU(2) Field: A Path Integral Approach
}

\author{
Sami Boudieb and Lyazid Chetouani \\ Département de Physique, Faculté des Sciences Exactes, Université Constantine 1, Constantine, Algeria \\ Correspondence should be addressed to Lyazid Chetouani; lyazidchetouani@gmail.com \\ Received 16 April 2013; Accepted 8 May 2013 \\ Academic Editors: G. Bonvicini, F.-H. Liu, L. Marek-Crnjac, and O. A. Sampayo \\ Copyright (c) 2013 S. Boudieb and L. Chetouani. This is an open access article distributed under the Creative Commons Attribution \\ License, which permits unrestricted use, distribution, and reproduction in any medium, provided the original work is properly cited. \\ The Green function for a Dirac particle moving in a non-Abelian SU(2) field and having a particular form is exactly determined by \\ the path integral approach. The wave functions were deduced from the residues of Green's function. It is shown that the classical \\ paths contributed mainly to the determination of the Green function.
}

\section{Introduction}

In the present paper, we suggest to determine the Green function for Dirac particle of mass $M$ in a $S U(2)$ non-Abelian field by the path integral approach. The $S U(2)$ non-Abelian field in question has the form $[1,2]$

$$
A_{\mu}^{a}=\left[\left(k_{1} x\right) b m^{a}+\left(k_{2} x\right) c m^{a}+h^{a}\right] k_{\mu},
$$

where

(i) $k_{1}, k_{2}$, and $k$ are, respectively, the polarizations of color wave and the isotropic wave such as

$$
k k_{1}=k k_{2}=k_{1} k_{2}=0,
$$

(ii) $b=b(k x), c=c(k x)$ are functions which depend only of $\varphi=k x$,

(iii) $m^{a}$ are generators of $S U(2)$ group which satisfy the condition

$$
m^{a}(\varphi) \cdot m^{a}(\varphi)=1,
$$

(iv) $h^{a}$ is a gauge function [3] such as

$$
h^{a}=\frac{1}{g} \varepsilon^{a b c} \frac{d m^{b}(\varphi)}{d \varphi} m^{c}(\varphi)=\frac{1}{g} \varepsilon^{a b c} m^{\prime b}(\varphi) m^{c}(\varphi)
$$

(v) and $a=1,2,3$ is a color index.
With this form of field, the Lorentz condition is satisfied:

$$
\partial^{\mu} A_{\mu}^{a}=0
$$

From the point of view of the literature, we can notice that there exists only one limiting number of cases of interactions where the equation of Dirac has analytical and exact solution. We can cite, for example, the simple cases such as the constant field [4], the plane wave $[5,6]$, the crossed electric and magnetic fields $[7,8]$, and the combination of constant homogeneous electromagnetic field with a plane wave field $[9,10]$. With the path integral formalism, we can cite [11-14], where the interactions have classical form.

For the non-Abelian case, at our knowledge, there exist no explicit calculations of propagators by the path integral formalism, except the formalism given by Fradkin et al. [15] and which we use in the present paper.

Note that for this problem treated in [1] the wave functions for a particle of spin $1 / 2$ are determined by solving the Yang-Mills equation.

Also, in this paper we propose to consider the nonAbelian field described previously in order to determine analytically Green's function by using the approach of path integral, to extract then the wave functions. 


\section{Green's Function for Dirac Particle: Local Approach}

First, let us proceed as indicated in the book [15] by replacing the generators of $S U(2)$ by a bilinear expression

$$
m_{a}=\frac{1}{4} \Gamma_{\alpha} t_{a \beta}^{\alpha} \Gamma_{\beta}
$$

where $t_{a \beta}^{\alpha}$ are traceless Hermitian matrices and $\Gamma_{\alpha}$ are the operators which satisfy the following anticommutation relations:

$$
\left\{\Gamma_{\alpha}, \Gamma_{\beta}\right\}=2 \delta_{\alpha \beta}
$$

First, let us write the equation satisfied by Green's function $S$ related to the Dirac particle submitted to the action of non-Abelian field [1]

$$
\begin{aligned}
& {\left[-(\gamma \widehat{p})+\frac{g}{8}(\gamma k)\left(b\left(k_{1} x\right)+c\left(k_{2} x\right)\right)\left(\Gamma_{\alpha} t_{\beta}^{\alpha} \Gamma_{\beta}\right)\right.} \\
& \left.\quad+\frac{g}{32 g}(\gamma k)\left(\frac{d \Gamma_{\alpha} t_{\beta}^{\alpha} \Gamma_{\beta}}{d \varphi}\left(\Gamma_{\alpha} t_{\beta}^{\alpha} \Gamma_{\beta}\right)\right)-M\right] \\
& \times S\left(x_{b}, x_{a}\right)=\delta^{4}\left(x_{b}-x_{a}\right),
\end{aligned}
$$

where $\gamma^{\mu}$ are usual Dirac matrices obeying to anticommutation relations

$$
\left\{\gamma^{\mu}, \gamma^{\nu}\right\}=2 g^{\mu \nu}, \quad \mu, \nu=0,1,2,3
$$

with $g^{\mu \nu}$ is the metric tensor of Minkowski space (diag $g_{\mu \nu}=$ $(1,-1,-1,-1))$.

In order to determine the Green function, we follow the usual construction method [11] of path integral form.

First, we know that the kernel

$$
S\left(x_{b}, x_{a}\right)=\left\langle x_{b}|\widehat{S}| x_{a}\right\rangle
$$

is a matrix element of operator $\widetilde{S}$, the solution of the following symbolic equation:

$$
\begin{aligned}
& \left(-(\gamma \widehat{p})+\frac{g}{8}(\gamma k)\left(b\left(k_{1} x\right)+c\left(k_{2} x\right)\right)\right. \\
& \times\left(\Gamma_{\alpha} t_{\beta}^{\alpha} \Gamma_{\beta}\right)+\frac{1}{32 g}(\gamma k) \\
& \left.\times\left(\frac{d \Gamma_{\alpha} t_{\beta}^{\alpha} \Gamma_{\beta}}{d \varphi}\left(\Gamma_{\alpha} t_{\beta}^{\alpha} \Gamma_{\beta}\right)\right)-M\right) \widehat{S}=I,
\end{aligned}
$$

or

$$
\begin{aligned}
\widehat{S}=I \times(( & -(\tilde{\gamma} \cdot \widehat{p})+\frac{g}{8}(\tilde{\gamma} \cdot k) \\
& \times\left(b\left(k_{1} \cdot x\right)+c\left(k_{2} \cdot x\right)\right)\left(\Gamma_{\alpha} t_{\beta}^{\alpha} \Gamma_{\beta}\right) \\
& +\frac{(\tilde{\gamma} \cdot k)}{32 g}\left(\frac{d \Gamma_{\alpha} t_{\beta}^{\alpha} \Gamma_{\beta}}{d \varphi}\left(\Gamma_{\alpha} t_{\beta}^{\alpha} \Gamma_{\beta}\right)\right) \\
& \left.\left.-M \gamma^{5}\right)\right)^{-1} \gamma^{5}=\widetilde{S} \gamma^{5},
\end{aligned}
$$

where $\gamma^{5}$ is introduced in order to homogenize the variables

$$
\gamma^{5}=\gamma^{0} \gamma^{1} \gamma^{2} \gamma^{3}, \quad\left(\gamma^{5}\right)^{2}=-1
$$

So, $\widetilde{S}$ can be written in the exponential form

$$
\widetilde{S}=\int_{0}^{\infty} d \lambda \int d \chi \int \exp \left(-i \lambda \widehat{H}_{l}(\chi, \widehat{p}, \widehat{x})\right)
$$

where $\lambda$ and $\chi$ are, respectively, bosonic and fermionic variables $[16,17]$. The Grassmann variables have the following properties:

$$
\chi^{2}=0, \quad \int d \chi=0, \quad \int \chi d \chi=1
$$

and the Hamiltonian system which governs the movement of the Dirac particle in the non-Abelian field is given by

$$
\begin{aligned}
& H_{l}= p^{2}-M^{2} \\
&-\frac{1}{16}((\widehat{p} \cdot \widehat{k}) \\
& \\
& \quad \times\left(\sigma\left(\frac{d \Gamma_{\alpha} t_{\beta}^{\alpha} \Gamma_{\beta}}{d \varphi}\left(\Gamma_{\alpha} t_{\beta}^{\alpha} \Gamma_{\beta}\right)\right)\right) \\
&+(\widehat{p} \widehat{k})\left(b\left(k_{1} x\right)+c\left(k_{2} x\right)\right)
\end{aligned}
$$




$$
\begin{gathered}
\left.\times\left(\sigma\left(\frac{1}{4} \Gamma_{\alpha} t_{\beta}^{\alpha} \Gamma_{\beta}\right)\right)\right)-i g(\widehat{k} \sigma \widehat{k}) \\
\times\left(\left(\frac{1}{32 g} \frac{d \Gamma_{\alpha} t_{\beta}^{\alpha} \Gamma_{\beta}}{d \varphi}\left(\Gamma_{\alpha} t_{\beta}^{\alpha} \Gamma_{\beta}\right)\right)\right. \\
\left.+\left\{\left(b\left(k_{1} x\right)+c\left(k_{2} x\right)\right)\left(\frac{1}{8} \Gamma_{\alpha} t_{\beta}^{\alpha} \Gamma_{\beta}\right)\right\}^{\prime}\right) \\
+\chi\left(-\widehat{p}+\frac{g}{2} \widehat{k}\left(b\left(k_{1} x\right)+c\left(k_{2} x\right)\right)\right. \\
\times\left(\sigma\left(\frac{1}{4} \Gamma_{\alpha} t_{\beta}^{\alpha} \Gamma_{\beta}\right)\right)+\frac{\widehat{k}}{2} \\
\left.\times\left(\sigma\left(\frac{1}{16 g} \frac{d \Gamma_{\alpha} t_{\beta}^{\alpha} \Gamma_{\beta}}{d \varphi}\left(\Gamma_{\alpha} t_{\beta}^{\alpha} \Gamma_{\beta}\right)\right)\right)-M \gamma^{5}\right) .
\end{gathered}
$$

In the configuration space, the Green function is [4]

$$
\widetilde{S}\left(x_{b}, x_{a}\right)=\int_{0}^{\infty} d \lambda \int d \chi \int\left\langle x_{b}\left|\exp \left(-i \lambda \widehat{H}_{l}(\chi, \widehat{p}, \hat{x})\right)\right| x_{a}\right\rangle,
$$

and the path integral formulation consists of

(i) subdividing the interval $\left[x_{a}, x_{b}\right]$ into $N$ equals intervals of length $\Delta \tau=\lambda / N$,

(ii) inserting the projectors

$$
\begin{gathered}
\int d^{4} x|x\rangle\left\langle x\left|=1, \quad d^{4} p\right| p\right\rangle\langle p|=1, \\
\int\langle x \mid p\rangle=\frac{1}{(2 \pi)^{4}} \exp (i p x),
\end{gathered}
$$

by using the actions on kets

$$
\hat{x}^{\mu}|x\rangle=x^{\mu}|x\rangle, \quad \hat{p}^{\mu}|p\rangle=p^{\mu}|p\rangle,
$$

in order to eliminate the operators $\widehat{x}, \widehat{p}$.
So the Green function can be written (with the midpoint prescription) as following

$$
\begin{aligned}
& G^{c}\left(x_{b}, x_{a}\right) \\
& =i T_{1} T_{2} \int_{0}^{\infty} d \lambda \int D x \int D p \times \exp i \\
& \times \int_{0}^{\lambda} d \tau\left[p \dot{x}+\left(p^{2}-M^{2}\right)-\frac{1}{16}\right. \\
& \times\left[(\widehat{p} \widehat{k})\left(\sigma\left(\frac{d \Gamma_{\alpha} t_{\beta}^{\alpha} \Gamma_{\beta}}{d \varphi}\left(\Gamma_{\alpha} t_{\beta}^{\alpha} \Gamma_{\beta}\right)\right)\right)\right. \\
& +(\widehat{p} \hat{k})\left(b\left(k_{1} x\right)+c\left(k_{2} x\right)\right) \\
& \left.\times\left(\sigma\left(\frac{1}{4} \Gamma_{\alpha} t_{\beta}^{\alpha} \Gamma_{\beta}\right)\right)\right] \\
& -i g(\widehat{k} \sigma \widehat{k})\left(\left(\frac{1}{32 g} \frac{d \Gamma_{\alpha} t_{\beta}^{\alpha} \Gamma_{\beta}}{d \varphi}\left(\Gamma_{\alpha} t_{\beta}^{\alpha} \Gamma_{\beta}\right)\right)\right. \\
& +\left\{\left(b\left(k_{1} x\right)+c\left(k_{2} x\right)\right)\right. \\
& \left.\left.\times\left(\frac{1}{8} \Gamma_{\alpha} t_{\beta}^{\alpha} \Gamma_{\beta}\right)\right\}^{\prime}\right) \\
& +\chi\left(-\widehat{p}+\frac{g}{2} \widehat{k}\left(b\left(k_{1} \cdot x\right)+c\left(k_{2} \cdot x\right)\right)\right. \\
& \times\left(\sigma \cdot\left(\frac{1}{4} \Gamma_{\alpha} t_{\beta}^{\alpha} \Gamma_{\beta}\right)\right)+\frac{\widehat{k}}{2} \\
& \times\left(\sigma \cdot\left(\frac{1}{16 g} \frac{d \Gamma_{\alpha} t_{\beta}^{\alpha} \Gamma_{\beta}}{d \varphi}\left(\Gamma_{\alpha} t_{\beta}^{\alpha} \Gamma_{\beta}\right)\right)\right) \\
& \left.\left.-M \gamma^{5}\right)\right] \text {, }
\end{aligned}
$$

where the $T_{1} T_{2}$-products are introduced because of the presence of matrices $\gamma^{\mu}$ and operators $\Gamma$ which do not commutate. To eliminate the $T_{1} T_{2}$-products, we used the following form path integral:

$$
\begin{gathered}
T_{1} \exp \left\{\int_{0}^{\lambda}\left(\rho_{1} \cdot \gamma\right) d \tau\right\} \\
=\exp \left(i \gamma \frac{\delta_{L}}{\delta \theta}\right)
\end{gathered}
$$




$$
\begin{gathered}
\times \int_{E_{1}} D \Psi \exp \left\{\int_{0}^{\lambda}\left((\Psi \cdot \dot{\Psi})-2 i\left(\rho_{1} \cdot \Psi\right)\right) d \tau\right. \\
+(\Psi(\lambda) \cdot \Psi(0))\}\left.\right|_{\Psi_{0}+\Psi_{1}=\theta},
\end{gathered}
$$

$$
\begin{aligned}
& T_{2} \exp \left\{\int_{0}^{\lambda}\left(\rho_{2} \cdot \Gamma\right) d \tau\right\} \\
& =\exp \left(i \Gamma \frac{\delta_{L}}{\delta \vartheta}\right) \\
& \times \int_{E_{2}} D \Upsilon \exp \left\{\int_{0}^{\lambda}\left((\Upsilon \cdot \dot{\Upsilon})-2 i\left(\rho_{2} \cdot \Upsilon\right)\right) d \tau\right. \\
& +(\Upsilon(\lambda) \cdot \Upsilon(0))\}\left.\right|_{\Upsilon_{0}+\Upsilon_{1}=\vartheta},
\end{aligned}
$$

$E_{1}=\{\Psi(\tau)$ where $\Psi(0)+\Psi(\lambda)=\theta\}, E_{2}=\{\Upsilon(\tau)$ where $\Upsilon(0)+$ $\Upsilon(\lambda)=9\}$ which are the domains of integration. $\rho_{1}, \rho_{2}$ are coupled currents to matrices $\gamma$ and operators $\Gamma$, and $\theta, \vartheta$ are Grassmann variables.

So, we obtain

$$
\begin{aligned}
& \widetilde{S}\left(x_{b}, x_{a}\right) \\
& =\exp \left(i \gamma \frac{\delta_{L}}{\delta \theta}\right) \exp \left(i \Gamma \frac{\delta_{L}}{\delta \vartheta}\right) \\
& \times \int_{0}^{\infty} d \lambda \int d \chi \int D x \int D p \int_{E_{1}} D \Psi \int_{E_{2}} D Y \\
& \times \exp \left[i \int_{0}^{\lambda} d \tau\right. \\
& \times\left[p \dot{x}+\left(p^{2}-M^{2}-\frac{i}{16}(p \Psi)(k \Psi)\right.\right. \\
& \times\left(t_{\beta}^{\alpha} \Upsilon_{\alpha}^{\prime} \Upsilon_{\beta}\right)-i \frac{g}{4}(p \Psi)(k \Psi) \\
& \left.\times\left(b\left(k_{1} x\right)+c\left(k_{2} x\right)\right)\left(t_{\beta}^{\alpha} \Upsilon_{\alpha} \Upsilon_{\beta}\right)\right) \\
& +\chi\left(-(p \Psi)+\frac{i}{32}(k \Psi)\left(\Psi\left(t_{\beta}^{\alpha} \Upsilon_{\alpha}^{\prime} \Upsilon_{\beta}\right)\right)\right. \\
& +i \frac{g}{8}(k \Psi)\left(b\left(k_{1} x\right)+c\left(k_{2} x\right)\right)
\end{aligned}
$$

$$
\begin{array}{r}
\left.\times\left(t_{\beta}^{\alpha} \Upsilon_{\alpha} \Upsilon_{\beta}\right)-M \Psi^{5}\right) \\
-i \Psi \dot{\Psi}-i \Upsilon \dot{\Upsilon}]+\Psi(\lambda) \Psi(0)
\end{array}
$$$$
+\Upsilon(\lambda) \Upsilon(0)]\left.\right|_{\theta=9=0},
$$

which is the Green function to calculate in the local approach of path integral.

Having obtained the path integral form for the Green function $\widetilde{S}$, let us perform the integrations.

We notice that the field depends on products $k x$ and $k_{i} x$, with $i=1,2$. First, we put $\varphi=k x$ and $\varphi_{i}=k_{i} x$, and then the variables $\varphi$ and $\varphi_{i}$ are rendered, respectively, independent of $k x$ and of $k_{i} x$ by introducing the following three identities $[11,12]$ :

$$
\begin{aligned}
& \int d \varphi_{b} \int d \varphi_{a} \delta\left(\varphi_{a}-k x_{a}\right) \int D \varphi \\
& \times \int D p_{\varphi} \exp \left\{i \int_{0}^{\lambda} p_{\varphi}(\dot{\varphi}-k \dot{x}) d \tau\right\}=1, \\
& \int d \varphi_{i b} \int d \varphi_{i a} \delta\left(\varphi_{i a}-k_{i} x_{a}\right) \int D \varphi_{i} \\
& \times \int D p_{\varphi_{i}} \exp \left\{i \int_{0}^{\lambda} p_{\varphi_{i}}\left(\dot{\varphi}_{i}-k_{i} \dot{x}\right) d \tau\right\}=1 .
\end{aligned}
$$

Then, $\widetilde{S}$ is rewritten as

$$
\begin{aligned}
\widetilde{S}\left(x_{b}, x_{a}\right) & \\
= & \exp \left(i \gamma \frac{\delta_{L}}{\delta \theta}\right) \exp \left(i \Gamma \frac{\delta_{L}}{\delta \vartheta}\right) \\
& \times \int_{0}^{\infty} d \lambda \int d \chi \int D x \int D p \\
& \times \int d \varphi_{b} \int d \varphi_{a} \delta\left(\varphi_{a}-k x_{a}\right) \int D \varphi \int D p_{\varphi} \\
& \times \prod_{i=1}^{2} \int d \varphi_{i b} \int d \varphi_{i a} \delta\left(\varphi_{i a}-k_{i} x_{a}\right) \int D \varphi_{i} \\
& \times \int_{\varphi_{i}} D \Psi \int_{E_{1}} D \Upsilon \\
& \times \exp i \int_{0}^{\lambda} d \tau\left[\left(p^{2}-M^{2}-i \frac{g}{32}(p \Psi)(k \cdot \Psi)\left(t_{\beta}^{\alpha} \Upsilon_{\alpha}^{\prime} \Upsilon_{\beta}\right)\right.\right. \\
& +\left(p-k p_{\varphi}-k_{1} p_{\varphi_{1}}-k_{2} p_{\varphi_{2}}\right) \dot{x} \\
& +p_{\varphi} \dot{\varphi}+p_{\varphi_{1}} \dot{\varphi}_{1}+p_{\varphi_{2}} \dot{\varphi}_{2}
\end{aligned}
$$




$$
\begin{aligned}
& \left.-i g(p \Psi)(k \Psi)\left(b \varphi_{1}+c \varphi_{2}\right)\left(t_{\beta}^{\alpha} \Upsilon_{\alpha} \Upsilon_{\beta}\right)\right) \\
& -\chi\left[(p \Psi)-\frac{i g}{2}(k \Psi)\left(\Psi\left(t_{\beta}^{\alpha} \Upsilon_{\alpha}^{\prime} \Upsilon_{\beta}\right)\right)\right. \\
& -i \frac{g}{2}(k \Psi)\left(b \varphi_{1}+c \varphi_{2}\right) \\
& \left.\times\left(t_{\beta}^{\alpha} \Upsilon_{\alpha} \Upsilon_{\beta}\right)+M \Psi^{5}\right] \\
& -i \Psi \dot{\Psi}-i \Upsilon \dot{\Upsilon}] \\
& +\Psi(\lambda) \Psi(0)+\Upsilon(\lambda) \Upsilon(0)]\left.\right|_{\theta=\vartheta=0}
\end{aligned}
$$

Using a shift on $p$ defined by

$$
p-k p_{\varphi}-k_{1} p_{\varphi_{1}}-k_{2} p_{\varphi_{2}} \longrightarrow p
$$

we obtain

$$
\begin{aligned}
& \widetilde{S}\left(x_{b}, x_{a}\right) \\
& =\exp \left(i \gamma \frac{\delta_{L}}{\delta \theta}\right) \exp \left(i \Gamma \frac{\delta_{L}}{\delta \vartheta}\right) \\
& \times \int_{0}^{\infty} d \lambda \int d \chi \int D x \int D p \\
& \times \int d \varphi_{b} \int d \varphi_{a} \delta\left(\varphi_{a}-k x_{a}\right) \int D \varphi \int D p_{\varphi} \\
& \times \prod_{i=1}^{2} \int d \varphi_{i b} \int d \varphi_{i a} \delta\left(\varphi_{i a}-k_{i} x_{a}\right) \\
& \times \int D \varphi_{i} \int D p_{\varphi_{i}} \int_{E_{1}} D \Psi \int_{E_{2}} D \Upsilon \\
& \times \exp \left[i \int_{0}^{\lambda} d \tau\right. \\
& \times\left[\left(p \dot{x}+p^{2}-M^{2}\right.\right. \\
& -i \frac{g}{32}(p \Psi)(k \Psi)\left(t_{\beta}^{\alpha} \Upsilon_{\alpha}^{\prime} \Upsilon_{\beta}\right) \\
& \left.-i g(p \Psi)(k \Psi)\left(b \varphi_{1}+c \varphi_{2}\right)\left(t_{\beta}^{\alpha} \Upsilon_{\alpha} \Upsilon_{\beta}\right)\right) \\
& +p_{\varphi}(\dot{\varphi}+2 k p)+p_{\varphi_{1}}\left(\dot{\varphi}_{1}+2 k_{1} p\right) \\
& +p_{\varphi_{2}}\left(\dot{\varphi}_{2}+2 k_{2} p\right)
\end{aligned}
$$

$$
\begin{gathered}
-\chi\left[(p \Psi)-\frac{i g}{2}(k \Psi)\left(\Psi\left(t_{\beta}^{\alpha} \Upsilon_{\alpha}^{\prime} \Upsilon_{\beta}\right)\right)\right. \\
-i \frac{g}{2}(k \Psi)\left(b \varphi_{1}+c \varphi_{2}\right)\left(t_{\beta}^{\alpha} \Upsilon_{\alpha} \Upsilon_{\beta}\right) \\
\left.\left.+M \Psi^{5}\right]-i \Psi \dot{\Psi}-i \Upsilon \dot{\Upsilon}\right] \\
+\Psi(\lambda) \Psi(0)+\Upsilon(\lambda) \Upsilon(0)]\left.\right|_{\theta=9=0},
\end{gathered}
$$

where $b=b(\varphi), c=c(\varphi)$.

If we integrate by part the first term in the action and then integrate the variables $x(\tau)$, a Dirac function $\delta(\dot{p})$ appeared, and the integrations on all the $p_{n}$ reduce $\int D p$ to a simple integral $\int d^{4} p /(2 \pi)^{4}$; that is, the momentum of the particle is conserved (constant) during the movement.

Thus, we have

$$
\widetilde{S}\left(x_{b}, x_{a}\right)
$$

$$
\begin{gathered}
=\exp \left(i \gamma \frac{\delta_{L}}{\delta \theta}\right) \exp \left(i \Gamma \cdot \frac{\delta_{L}}{\delta \vartheta}\right) \\
\times \int_{0}^{\infty} d \lambda \int d \chi \int \frac{d^{4} p}{(2 \pi)^{4}} \\
\times \exp \left\{i p\left(x_{b}-x_{a}\right)+i \lambda\left(p^{2}-M^{2}\right)\right\} \\
\times \int d \varphi_{b} \int d \varphi_{a} \delta\left(\varphi_{a}-k x_{a}\right) \int D \varphi \int D p_{\varphi} \\
\times \prod_{i=1}^{2} \int d \varphi_{i b} \int d \varphi_{i a} \delta\left(\varphi_{i a}-k_{i} x_{a}\right) \\
\times \int D \varphi_{i} \int D p_{\varphi_{i}} \int_{E_{1}} D \Psi \int_{E_{2}} D \Upsilon \\
\times \exp \left[i \int_{0}^{\lambda} d \tau\right. \\
\times\left[\left(-i \frac{g}{32}(p \Psi)(k \Psi)\left(t_{\beta}^{\alpha} \Upsilon_{\alpha}^{\prime} \Upsilon_{\beta}\right)\right.\right. \\
\left.\quad-i g(p \Psi)(k \Psi)\left(b \varphi_{1}+c \varphi_{2}\right)\left(t_{\beta}^{\alpha} \Upsilon_{\alpha} \Upsilon_{\beta}\right)\right) \\
+p_{\varphi}(\dot{\varphi}+2 k p)+p_{\varphi_{1}}\left(\dot{\varphi}_{1}+2 k_{1} p\right) \\
+p_{\varphi_{2}}\left(\dot{\varphi}_{2}+2 k_{2} p\right) \\
-\chi\left[(p \Psi)-\frac{i g}{2}(k \Psi)\left(\Psi\left(t_{\beta}^{\alpha} \Upsilon_{\alpha}^{\prime} \Upsilon_{\beta}\right)\right)\right.
\end{gathered}
$$




$$
\begin{aligned}
& -i \frac{g}{2}(k \Psi)\left(b \varphi_{1}+c \varphi_{2}\right) \\
& \left.\left.\times\left(t_{\beta}^{\alpha} \Upsilon_{\alpha} \Upsilon_{\beta}\right)+M \Psi^{5}\right]-i \Psi \dot{\Psi}-i \Upsilon \dot{\Upsilon}\right] \\
& +\Psi(\lambda) \Psi(0)+\Upsilon(\lambda) \Upsilon(0)]\left.\right|_{\theta=9=0} \cdot
\end{aligned}
$$

We notice also, that there is dependence of product of $k \Psi_{a}$ in the action. So if we put $\xi_{a}=k \Psi_{a}$, these new variables are rendered independent $[11,12]$ by the introduction of the following relation:

$$
\begin{aligned}
& \int d \xi_{a} d \xi_{b} \delta\left(\xi_{a}-\left(k \cdot \Psi_{a}\right)\right) \\
& \times \int D p_{\xi} \int D \xi \exp \left\{i \int_{0}^{\lambda} p_{\xi}(\dot{\xi}-(k \cdot \dot{\Psi})) d \tau\right\}=1,
\end{aligned}
$$

with $\xi$ and $p_{\xi}$ being Grassmann variables.

Then $\widetilde{S}$, with these new variables, is rewritten as

$$
\begin{aligned}
& \widetilde{S}\left(x_{b}, x_{a}\right) \\
& =\exp \left(i \gamma \frac{\delta_{L}}{\delta \theta}\right) \exp \left(i \Gamma \frac{\delta_{L}}{\delta \vartheta}\right) \\
& \times \int_{0}^{\infty} d \lambda \int d \chi \int \frac{d^{4} p}{(2 \pi)^{4}} \\
& \times \exp \left\{i p\left(x_{b}-x_{a}\right)+i \lambda\left(p^{2}-M^{2}\right)\right\} \\
& \times \int d \varphi_{b} \int d \varphi_{a} \delta\left(\varphi_{a}-k x_{a}\right) \int D \varphi \int D p_{\varphi} \\
& \times \prod_{i=1}^{2} \int d \varphi_{i b} \int d \varphi_{i a} \delta\left(\varphi_{i a}-k_{i} x_{a}\right) \int D \varphi_{i} \\
& \times \int D p_{\varphi_{i}} \int d \xi_{a} d \xi_{b} \delta\left(\xi_{a}-\left(k \cdot \Psi_{a}\right)\right) \\
& \times \int D \xi \int D p_{\xi} \int_{E_{1}} D \Psi \int_{E_{2}} D \Upsilon \\
& \times \exp \left[i \int_{0}^{\lambda} d \tau\right. \\
& \times\left[\left(-i \frac{g}{32}(p \Psi)(k \Psi)\left(t_{\beta}^{\alpha} \Upsilon_{\alpha}^{\prime} \Upsilon_{\beta}\right)\right.\right. \\
& \left.-i g(p \Psi)(k \Psi)\left(b \varphi_{1}+c \varphi_{2}\right)\left(t_{\beta}^{\alpha} \Upsilon_{\alpha} \Upsilon_{\beta}\right)\right) \\
& +p_{\varphi}(\dot{\varphi}+2 k p)+p_{\varphi_{1}}\left(\dot{\varphi}_{1}+2 k_{1} p\right)
\end{aligned}
$$

$$
\begin{gathered}
+p_{\varphi_{2}}\left(\dot{\varphi}_{2}+2 k_{2} p\right)+p_{\xi}(\dot{\xi}-(k \dot{\Psi})) \\
-\chi\left[(p \Psi)-\frac{i g}{2}(k \Psi)\left(\Psi\left(t_{\beta}^{\alpha} \Upsilon_{\alpha}^{\prime} \Upsilon_{\beta}\right)\right)\right. \\
-i \frac{g}{2}(k \Psi)\left(b \varphi_{1}+c \varphi_{2}\right)\left(t_{\beta}^{\alpha} \Upsilon_{\alpha} \Upsilon_{\beta}\right) \\
\left.\left.+M \Psi^{5}\right]-i \Psi \dot{\Psi}-i \Upsilon \dot{\Upsilon}\right] \\
+\Psi(\lambda) \Psi(0)+\Upsilon(\lambda) \Upsilon(0)]\left.\right|_{\theta=9=0} .
\end{gathered}
$$

Let us remark that the integrations over the Grassmann variables $\Psi$ are subjected to the boundary conditions. We can render free these integrations, if we use new variables $\omega$ (velocities) defined by $[15,18]$

$$
\omega(\tau)=\frac{d \Psi(\tau)}{d \tau}
$$

or by integration we have the relation

$$
\Psi(\tau)=\frac{1}{2} \int_{0}^{\lambda} \varepsilon\left(\tau-\tau^{\prime}\right) \omega\left(\tau^{\prime}\right) d \tau^{\prime}+\frac{\theta}{2}
$$

between $\Psi$ and $\omega$.

Since

$$
\begin{aligned}
& \int_{0}^{\lambda} d \tau \Psi \dot{\Psi}+\Psi(\lambda) \Psi(0) \\
& \quad=\int_{0}^{\lambda} \int_{0}^{\lambda^{\prime}} d \tau d \tau^{\prime} \omega(\tau) \varepsilon\left(\tau-\tau^{\prime}\right) \omega\left(\tau^{\prime}\right) \\
& \quad=-\frac{1}{2} \omega * \varepsilon * \omega, \\
& \int_{E} D \Psi=\int_{E} D \omega,
\end{aligned}
$$

where the following notation is used:

$$
a * b * c=\iint a(\tau) b\left(\tau, \tau^{\prime}\right) c\left(\tau^{\prime}\right) d \tau d \tau^{\prime}
$$

we obtain for $\widetilde{S}$

$$
\begin{aligned}
& \widetilde{S}\left(x_{b}, x_{a}\right) \\
& =\exp \left(i \gamma \frac{\delta_{L}}{\delta \theta}\right) \exp \left(i \Gamma \frac{\delta_{L}}{\delta \vartheta}\right) \\
& \quad \times \int_{0}^{\infty} d \lambda \int d \chi \int \frac{d^{4} p}{(2 \pi)^{4}}
\end{aligned}
$$




$$
\begin{gathered}
\times \exp \left\{i p\left(x_{b}-x_{a}\right)+i \lambda\left(p^{2}-M^{2}\right)\right\} \\
\times \int d \varphi_{b} \int d \varphi_{a} \delta\left(\varphi_{a}-k x_{a}\right) \int D \varphi \int D p_{\varphi} \\
\times \prod_{i=1}^{2} \int d \varphi_{i b} \int d \varphi_{i a} \delta\left(\varphi_{i a}-k_{i} x_{a}\right) \int D \varphi_{i} \int D p_{\varphi_{i}} \\
\times \int d \xi_{a} \int d \xi_{b} \int D p_{\xi} \int D \xi \\
\times \int_{E_{1}} D \omega \delta\left(\xi_{a}+\frac{1}{2}(k(\omega-\theta))\right) \int_{E_{2}} D \Upsilon \\
\times \exp \left[i \int_{0}^{\lambda} d \tau\right. \\
\times\left(-\frac{i}{2} g p(\varepsilon * \omega) \xi\right. \\
-\frac{i}{2} g p(\varepsilon * \omega) \xi\left(b \varphi_{1}+c \varphi_{2}\right) \\
-\frac{i}{2} g(p \theta)\left(b \varphi_{1}+c \varphi_{2}\right) \xi+p_{\varphi}(\dot{\varphi}+2 k p) \\
+p_{\varphi_{1}}\left(\dot{\varphi}_{1}+2 k_{1} p\right)+p_{\varphi_{2}}\left(\dot{\varphi}_{2}+2 k_{2} p\right) \\
+p_{\xi} \dot{\xi}-i \Upsilon \dot{\Upsilon} \\
+\chi\left[-\frac{1}{2} p(\varepsilon * \omega)\right. \\
-\frac{1}{2}(p \theta)+\frac{i}{4} g(\varepsilon * \omega)\left(t_{\beta}^{\alpha} \Upsilon_{\alpha}^{\prime} \Upsilon_{\beta}\right) \\
+\frac{i}{4} g(\varepsilon * \omega) \xi\left(b \varphi_{1}+c \varphi_{2}\right) \\
+\frac{i}{4} g(\theta \xi)\left(b \varphi_{1}+c \varphi_{2}\right) \\
\left.\left.\times\left(t_{\beta}^{\alpha} \Upsilon_{\alpha} \Upsilon_{\beta}\right)+M\left(\varepsilon * \omega^{5}+\theta^{5}\right)\right]\right) \\
\left.\times \varepsilon+(\Upsilon(\lambda) \cdot \Upsilon(0))]\left.\right|_{\theta=9=0}\right)
\end{gathered}
$$

By using a shift for $\omega$

$$
\begin{aligned}
& \bar{\omega}(\tau) \longrightarrow \omega(\tau)+i k\left(\varepsilon^{-1} * p_{\xi}\right) \\
& \quad+i k_{1}\left(\varepsilon^{-1} * p_{\xi_{1}}\right)+i k_{2}\left(\varepsilon^{-1} * p_{\xi_{2}}\right),
\end{aligned}
$$

the Green function can be simplified and take the following form:

$$
\begin{aligned}
& \widetilde{S}\left(x_{b}, x_{a}\right) \\
& =\exp \left(i \gamma \frac{\delta_{L}}{\delta \theta}\right) \exp \left(i \Gamma \frac{\delta_{L}}{\delta \vartheta}\right)
\end{aligned}
$$

$\times \int_{0}^{\infty} d \lambda \int d \chi \int \frac{d^{4} p}{(2 \pi)^{4}}$

$\times \exp \left\{i p\left(x_{b}-x_{a}\right)+i \lambda\left(p^{2}-M^{2}\right)\right\}$

$\times \int d \varphi_{b} \int d \varphi_{a} \delta\left(\varphi_{a}-k x_{a}\right) \int D \varphi \int D p_{\varphi}$

$\times \prod_{i=1}^{2} \int d \varphi_{i b} \int d \varphi_{i a} \delta\left(\varphi_{i a}-k_{i} x_{a}\right)$

$\times \int D \varphi_{i} \int D p_{\varphi_{i}} \int d \xi_{a} \int d \xi_{b} \int D p_{\xi} \int D \xi$

$\times \int_{E_{1}} D \omega \delta\left(\xi_{a}+\frac{1}{2}(k \cdot(\omega-\theta))\right) \int_{E_{2}} D \Upsilon$

$\times \exp \left[i \int_{0}^{\lambda} d \tau\right.$

$$
\times\left(-\frac{i}{2} g\left(p \left(\varepsilon * \omega-i\left(k \cdot p_{\xi}\right)-i\left(k_{1} \cdot p_{\xi_{1}}\right)\right.\right.\right.
$$$$
\left.\left.-i\left(k_{2} \cdot p_{\xi_{2}}\right)\right) \xi\right)
$$$$
-\frac{i}{2} g p\left(\varepsilon * \omega-i\left(k \cdot p_{\xi}\right)-i\left(k_{1} \cdot p_{\xi_{1}}\right)\right.
$$$$
\left.-i\left(k_{2} \cdot p_{\xi_{2}}\right)\right)
$$$$
\times \xi\left(b \cdot \varphi_{1}+c \cdot \varphi_{2}\right)-\frac{i}{2} g(p \cdot \theta)
$$$$
\times\left(b \varphi_{1}+c \varphi_{2}\right) \xi+p_{\varphi}(\dot{\varphi}+2(k \cdot p))
$$$$
+p_{\varphi_{1}}\left(\dot{\varphi}_{1}+2 k_{1} p\right)+p_{\varphi_{2}}\left(\dot{\varphi}_{2}+2 k_{2} p\right)
$$$$
+p_{\xi} \dot{\xi}-i \Upsilon \dot{\Upsilon}
$$$$
+\chi\left[-\frac{1}{2} p\right.
$$$$
\times\left(\varepsilon * \omega-i\left(k p_{\xi}\right)-i\left(k_{1} p_{\xi_{1}}\right)-i\left(k_{2} p_{\xi_{2}}\right)\right)
$$$$
-\frac{1}{2}(p \theta)+\frac{i}{4} g
$$$$
\times\left(\varepsilon * \omega-i\left(k \cdot p_{\xi}\right)\right.
$$

$$
\left.-i\left(k_{1} p_{\xi_{1}}\right)-i\left(k_{2} p_{\xi_{2}}\right)\right)
$$$$
\times\left(t_{\beta}^{\alpha} \Upsilon_{\alpha}^{\prime} \Upsilon_{\beta}\right)+\frac{i}{4} g
$$$$
\times\left(\varepsilon * \omega-i\left(k p_{\xi}\right)-i\left(k_{1} p_{\xi_{1}}\right)-i\left(k_{2} p_{\xi_{2}}\right)\right)
$$$$
\times \xi\left(b \varphi_{1}+c \varphi_{2}\right)
$$$$
+\frac{i}{4} g(\theta \xi)\left(b \varphi_{1}+c \varphi_{2}\right)\left(t_{\beta}^{\alpha} \Upsilon_{\alpha} \Upsilon_{\beta}\right)
$$

$\left.\left.+M\left(\varepsilon * \omega^{5}+\theta^{5}\right)\right]\right)$ 


$$
\begin{aligned}
& -\frac{1}{2}\left(\omega * \varepsilon * \omega-2 i(k \omega) p_{\xi}\right. \\
& \left.\quad-2 i\left(k_{1} \omega\right) p_{\xi_{1}}-2 i\left(k_{1} \omega\right) p_{\xi_{2}}\right) \\
& +\Upsilon(\lambda) \Upsilon(0)]\left.\right|_{\theta=9=0} \cdot
\end{aligned}
$$

At this level, to integrate velocities $\omega$, first, we insert the integral representation of the Dirac function

$$
\begin{aligned}
& \delta\left(\xi_{a}+\frac{1}{2}(k(\omega-\theta))\right) \\
& \quad=\int d p_{\xi_{a}} \exp \left\{i p_{\xi_{a}}\left(\xi_{a}+\frac{1}{2}(k(\omega-\theta))\right)\right\},
\end{aligned}
$$

with $p_{\xi_{a}}$ being Grassmann variable; then we have

$$
\begin{aligned}
& \widetilde{S}\left(x_{b}, x_{a}\right) \\
& =\exp \left(i \gamma \frac{\delta_{L}}{\delta \theta}\right) \exp \left(i \Gamma \frac{\delta_{L}}{\delta \vartheta}\right) \\
& \times \int_{0}^{\infty} d \lambda \int d \chi \int \frac{d^{4} p}{(2 \pi)^{4}} \\
& \times \exp \left\{i p\left(x_{b}-x_{a}\right)+i \lambda\left(p^{2}-M^{2}\right)\right\} \\
& \times \int d \varphi_{b} \int d \varphi_{a} \delta\left(\varphi_{a}-k x_{a}\right) \int D \varphi \int D p_{\varphi} \\
& \times \prod_{i=1}^{2} \int d \varphi_{i b} \int d \varphi_{i a} \delta\left(\varphi_{i a}-k_{i} x_{a}\right) \int D \varphi_{i} \\
& \times \int D p_{\varphi_{i}} \int d \xi_{a} \int d p_{\xi_{a}} \int d \xi_{b} \int D p_{\xi} \int D \xi \\
& \times \int_{E_{1}} D \omega \delta\left(\xi_{a}+\frac{1}{2}(k(\omega-\theta))\right) \int_{E_{2}} D \Upsilon \\
& \times \exp \left[i \int_{0}^{\lambda} d \tau\right. \\
& \times\left(-\frac{i}{2} g\right. \\
& \times\left(p \left(\varepsilon * \omega-i\left(k p_{\xi}\right)-i\left(k_{1} p_{\xi_{1}}\right)\right.\right. \\
& \left.\left.-i\left(k_{2} p_{\xi_{2}}\right)\right) \xi\right) \\
& -\frac{i}{2} g p\left(\varepsilon * \omega-i\left(k p_{\xi}\right)\right. \\
& \left.-i\left(k_{1} p_{\xi_{1}}\right)-i\left(k_{2} p_{\xi_{2}}\right)\right)
\end{aligned}
$$

$$
\begin{aligned}
& \times \xi\left(b \varphi_{1}+c \varphi_{2}\right)-\frac{i}{2} g(p \theta)\left(b \varphi_{1}+c \varphi_{2}\right) \xi \\
& +p_{\varphi}(\dot{\varphi}+2 k p)+p_{\varphi_{1}}\left(\dot{\varphi}_{1}+2 k_{1} p\right) \\
& +p_{\varphi_{2}}\left(\dot{\varphi}_{2}+2 k_{2} p\right)+p_{\xi} \dot{\xi}+p_{\xi_{a}} \\
& \times\left(\xi_{a}+\frac{1}{2}(k(\omega-\theta))\right)-i \Upsilon \dot{\Upsilon}
\end{aligned}
$$$$
+\chi\left[-\frac{1}{2} p\left(\varepsilon * \omega-i\left(k p_{\xi}\right)\right.\right.
$$$$
\left.-i\left(k_{1} p_{\xi_{1}}\right)-i\left(k_{2} p_{\xi_{2}}\right)\right)
$$$$
-\frac{1}{2}(p \theta)+\frac{i}{4} g
$$$$
\times\left(\varepsilon * \omega-i\left(k p_{\xi}\right)\right.
$$$$
\left.-i\left(k_{1} p_{\xi_{1}}\right)-i\left(k_{2} p_{\xi_{2}}\right)\right)
$$$$
\times\left(t_{\beta}^{\alpha} \Upsilon_{\alpha}^{\prime} \Upsilon_{\beta}\right)+\frac{i}{4} g
$$$$
\times\left(\varepsilon * \omega-i\left(k p_{\xi}\right)\right.
$$$$
\left.-i\left(k_{1} p_{\xi_{1}}\right)-i\left(k_{2} p_{\xi_{2}}\right)\right)
$$$$
\times \xi\left(b \varphi_{1}+c \varphi_{2}\right)+\frac{i}{4} g(\theta \xi)\left(b \varphi_{1}+c \varphi_{2}\right)
$$$$
\left.\left.\times\left(t_{\beta}^{\alpha} \Upsilon_{\alpha} \Upsilon_{\beta}\right)+M\left(\varepsilon * \omega^{5}+\theta^{5}\right)\right]\right)
$$$$
-\frac{1}{2}\left(\omega * \varepsilon * \omega-2 i(k \omega) p_{\xi}\right.
$$$$
\left.-2 i\left(k_{1} \omega\right) p_{\xi_{1}}-2 i\left(k_{1} \omega\right) p_{\xi_{2}}\right)
$$$$
+\Upsilon(\lambda) \Upsilon(0)]\left.\right|_{\theta=9=0} .
$$

We notice that the integration over $\omega^{5}$ can be eliminated thanks to the following result:

$$
\int D \omega^{5} \exp \left\{-\frac{1}{2} \omega_{5} * \varepsilon * \omega^{5}-\frac{i M}{2} \varepsilon * \omega^{5} \chi\right\}=1
$$

and for other variables $\omega^{\mu}(\mu=0,1,2,3)$, the integrations have the following forms:

$$
\int D \omega \exp \left\{\int_{0}^{\lambda}\left[-\frac{1}{2} \omega_{\mu} \varepsilon \omega^{\mu}+L_{\mu} \omega^{\mu}\right] d \tau\right\}
$$


where

$$
\begin{aligned}
L_{\mu}(\tau)=-\frac{i g}{2} \int_{0}^{\lambda} p_{\mu} \xi(s)\left(1+\left(b \varphi_{1}+c \varphi_{2}\right)\right) \varepsilon(\tau-s) d s & +\chi \int_{0}^{\lambda}\left(-\frac{1}{2} p_{\mu}+\frac{i}{4} g k_{\mu}\right. \\
& \left.\times\left(\left(t_{\beta}^{\alpha} \Upsilon_{\alpha}^{\prime} \Upsilon_{\beta}\right)+\xi(s)\left(b \varphi_{1}+c \varphi_{2}\right)\right)\right) \\
& \times \varepsilon(\tau-s) d s+\frac{1}{2} k_{\mu} p_{\xi_{a}} .
\end{aligned}
$$

After we have integrated over the $\omega, \widetilde{S}$ is reduced to

$$
\begin{aligned}
& \widetilde{S}\left(x_{b}, x_{a}\right) \\
& =\exp \left(i \gamma \frac{\delta_{L}}{\delta \theta}\right) \exp \left(i \Gamma \frac{\delta_{L}}{\delta \vartheta}\right) \\
& \times \int_{0}^{\infty} d \lambda \int d \chi \\
& \times \int \frac{d^{4} p}{(2 \pi)^{4}} \exp \left\{i p\left(x_{b}-x_{a}\right)+i \lambda\left(p^{2}-M^{2}\right)\right\} \\
& \times \int d \varphi_{b} \int d \varphi_{a} \delta\left(\varphi_{a}-k x_{a}\right) \\
& \times \int D \varphi \int D p_{\varphi} \prod_{i=1}^{2} \int d \varphi_{i b} \\
& \times \int d \varphi_{i a} \delta\left(\varphi_{i a}-k_{i} x_{a}\right) \int D \varphi_{i} \int D p_{\varphi_{i}} \\
& \times \int d \xi_{a} \int d p_{\xi_{a}} \int d \xi_{b} \int D p_{\xi} \int D \xi \int_{E_{2}} D \Upsilon \\
& \times \exp \left[i \int_{0}^{\lambda} d \tau\right. \\
& \times\left(p_{\varphi}(\dot{\varphi}+2 k p)+p_{\varphi_{1}}\left(\dot{\varphi}_{1}+2 k_{1} p\right)\right. \\
& +p_{\varphi_{2}}\left(\dot{\varphi}_{2}+2 k_{2} p\right)+p_{\xi} \dot{\xi}+p_{\xi_{a}} \\
& \times\left(\xi_{a}-\frac{1}{2} k \theta\right)-i \dot{\Upsilon} \\
& +\chi\left[-\frac{1}{2}(p \theta)+\frac{i}{4} g\left(t_{\beta}^{\alpha} \Upsilon_{\alpha} \Upsilon_{\beta}\right)\right. \\
& +\frac{i}{4} g \xi\left(b \varphi_{1}+c \varphi_{2}\right)+\frac{i}{4} g(\theta \xi)\left(b \varphi_{1}+c \varphi_{2}\right) \\
& \left.\left.\times\left(t_{\beta}^{\alpha} \Upsilon_{\alpha} \Upsilon_{\beta}\right)+M \theta^{5}\right]\right) \\
& +\Upsilon(\lambda) \Upsilon(0)]\left.\right|_{\theta=9=0},
\end{aligned}
$$

and the integrations on variables $p_{\xi}$ give

$$
\dot{\xi}=0, \quad \text { that is, } \xi_{a}=\xi_{b}=c^{s t} .
$$

Thus, $\widetilde{S}$ becomes

$$
\begin{aligned}
& \widetilde{S}\left(x_{b}, x_{a}\right) \\
& =\exp \left(i \gamma \frac{\delta_{L}}{\delta \theta}\right) \exp \left(i \Gamma \frac{\delta_{L}}{\delta \vartheta}\right) \\
& \times \int_{0}^{\infty} d \lambda \int d \chi \\
& \times \int \frac{d^{4} p}{(2 \pi)^{4}} \exp \left\{i p\left(x_{b}-x_{a}\right)+i \lambda\left(p^{2}-M^{2}\right)\right\} \\
& \times \int d \varphi_{b} \int d \varphi_{a} \delta\left(\varphi_{a}-k x_{a}\right) \int D \varphi \int D p_{\varphi} \\
& \times \prod_{i=1}^{2} \int d \varphi_{i b} \int d \varphi_{i a} \delta\left(\varphi_{i a}-k_{i} x_{a}\right) \int D \varphi_{i} \\
& \times \int D p_{\varphi_{i}} \int d \xi_{a} \int d p_{\xi_{a}} \int D \xi \int_{E_{2}} D \Upsilon \\
& \times \exp \left[i \int_{0}^{\lambda} d \tau \quad+\frac{i}{4} g \xi\left(b \varphi_{1}+c \varphi_{2}\right)+\frac{i}{4} g(\theta \xi)\right. \\
& \times\left(p_{\varphi}(\dot{\varphi}+2(k p))\right. \\
& +p_{\varphi_{1}}\left(\dot{\varphi}_{1}+2 k_{1} p\right)+p_{\varphi_{2}}\left(\dot{\varphi}_{2}+2 k_{2} p\right) \\
& +p_{\xi_{a}}\left(\xi_{a}-\frac{1}{2} k \theta\right)-i \dot{\Upsilon} \\
& +\chi\left[-\frac{1}{2}(p \theta)+\frac{i}{4} g\left(t_{\beta}^{\alpha} \Upsilon_{\alpha}^{\prime} \Upsilon_{\beta}\right)\right. \\
& +\Upsilon(\lambda) \Upsilon(0)]\left.\right|_{\theta=9=0}
\end{aligned}
$$

The integrations over $p_{\xi_{a}}$ give the following result:

$$
\xi_{a}-\frac{1}{2}(k \theta)=0, \quad \text { that is, } \xi_{a}=\xi_{b}=\frac{1}{2}(k \theta),
$$

and the Green function is reduced to

$$
\begin{aligned}
& \widetilde{S}\left(x_{b}, x_{a}\right) \\
& =\exp \left(i \gamma \frac{\delta_{L}}{\delta \theta}\right) \exp \left(i \Gamma \frac{\delta_{L}}{\delta \vartheta}\right) \\
& \quad \times \int_{0}^{\infty} d \lambda \int d \chi \int \frac{d^{4} p}{(2 \pi)^{4}}
\end{aligned}
$$




$$
\begin{aligned}
& \times \exp \left\{i p\left(x_{b}-x_{a}\right)+i \lambda\left(p^{2}-M^{2}\right)\right\} \\
& \times \int d \varphi_{b} \int d \varphi_{a} \delta\left(\varphi_{a}-k x_{a}\right) \int D \varphi \int D p_{\varphi} \\
& \times \prod_{i=1}^{2} \int d \varphi_{i b} \int d \varphi_{i a} \delta\left(\varphi_{i a}-k_{i} x_{a}\right) \\
& \times \int D \varphi_{i} \int D p_{\varphi_{i}} \int d \xi_{a} \int D \xi \int_{E_{2}} D \Upsilon \\
& \times \exp \left[i \int_{0}^{\lambda} d \tau\right. \\
& \times\left(p_{\varphi}(\dot{\varphi}+2 k p)\right. \\
& +p_{\varphi_{1}}\left(\dot{\varphi}_{1}+2 k_{1} p\right) \\
& +p_{\varphi_{2}}\left(\dot{\varphi}_{2}+2 k_{2} p\right)-i \Upsilon \dot{\Upsilon} \\
& +\chi\left[-\frac{1}{2}(p \theta)+\frac{i}{4} g\left(t_{\beta}^{\alpha} \Upsilon_{\alpha}^{\prime} \Upsilon_{\beta}\right)\right. \\
& +\frac{i}{4} g \xi\left(b \varphi_{1}+c \varphi_{2}\right)+\frac{i}{4} g(\theta \xi) \\
& \left.\left.\times\left(b \varphi_{1}+c \varphi_{2}\right)\left(t_{\beta}^{\alpha} \Upsilon_{\alpha} \Upsilon_{\beta}\right)+M \theta^{5}\right]\right) \\
& +\Upsilon(\lambda) \Upsilon(0)]\left.\right|_{\theta=9=0}
\end{aligned}
$$

By integrations on $p_{\varphi}, p_{\varphi_{1}}$, and $p_{\varphi_{2}}$ again, three Dirac functions appear also

$$
\delta(\dot{\varphi}+2(k p)), \quad \delta\left(\dot{\varphi}_{i}+2\left(k_{i} p\right)\right), \quad(i=1,2) ;
$$

that is, the equations

$$
\frac{d \varphi}{d \tau}=-2(k p), \quad \frac{d \varphi_{i}}{d \tau}=-2\left(k_{i} p\right),
$$

for solutions

$$
\begin{gathered}
\varphi(\tau)=-2(k p) \tau+c^{t e}, \\
\varphi_{i}(\tau)=-2\left(k_{i} p\right) \tau+c^{t e}
\end{gathered}
$$

contribute to the determination of the Green function.

Now, we introduce the integral representations of three $\delta$ :

$$
\begin{aligned}
\delta\left(\varphi_{a}-\right. & \left.k x_{a}-(k p) \lambda\right) \\
= & \frac{1}{2 \pi} \int d p_{\varphi} \\
& \times \exp \left\{i p_{\varphi}\left(\varphi_{a}-k x_{a}-(k p) \lambda\right)\right\}, \\
\delta\left(\varphi_{i a}-k_{i} x_{a}-\left(k_{i} p\right) \lambda\right) & \\
= & \frac{1}{2 \pi} \int d p_{\varphi_{i}} \\
\quad & \times \exp \left\{i p_{\varphi_{i}}\left(\varphi_{i a}-k_{i} x_{a}-\left(k_{i} p\right) \lambda\right)\right\} .
\end{aligned}
$$

After having changed $p$ into $p-k p_{\varphi_{b}}-k_{1} p_{\varphi_{1 b}}-k_{2} p_{\varphi_{2 b}}$, the relations (22) are reused to reintroduce the generators $\Gamma_{\alpha}$ of $S U(2)$ group since there is no order problem; the $T_{2}$ product is omitted, then the Green function $\widetilde{S}$ symmetrized with respect to positions $x_{a}$ and $x_{b}$ takes the following form:

$$
\begin{aligned}
& \widetilde{S}\left(x_{b}, x_{a}\right) \\
& =\exp \left(i \gamma \frac{\delta_{L}}{\delta \theta}\right) \int_{0}^{\infty} d \lambda \int d \chi \\
& \times \int \frac{d^{4} p}{(2 \pi)^{4}} \exp \left\{i p\left(x_{b}-x_{a}\right)+i \lambda\left(p^{2}-M^{2}\right)\right\} \\
& \times \exp \left[i \int_{\varphi_{a}}^{\varphi_{b}} d \varphi(k \theta)\right. \\
& \times\left(-\frac{i g}{8(k p)}(k p)\right. \\
& \times\left(\frac{d \Gamma_{\alpha} t_{\beta}^{\alpha} \Gamma_{\beta}}{d \varphi}\left(\Gamma_{\alpha} t_{\beta}^{\alpha} \Gamma_{\beta}\right)\right)-\frac{1}{4(k p)^{2}} \\
& \times \frac{p^{2}}{(1-(1 / 2) \chi)^{2}} \chi
\end{aligned}
$$$$
\left.-\frac{i g}{16}\left(\frac{d \Gamma_{\alpha} t_{\beta}^{\alpha} \Gamma_{\beta}}{d \varphi}\left(\Gamma_{\alpha} t_{\beta}^{\alpha} \Gamma_{\beta}\right)\right) \chi\right)
$$

$$
\begin{aligned}
&-\frac{i}{2}\left((p \theta)+M \theta^{5}\right) \chi \\
&+\frac{(k \theta)}{(k p)}\left(-\frac{1}{32} \int_{\varphi_{a}}^{\varphi_{b}} d \varphi\left(\frac{d\left(\Gamma_{\alpha} t_{\beta}^{\alpha} \Gamma_{\beta}\right)}{d \varphi}\left(\Gamma_{\alpha} t_{\beta}^{\alpha} \Gamma_{\beta}\right)\right)\right. \\
& \times(k \vartheta) \chi+\frac{k^{2}}{128} \int_{\varphi_{a}}^{\varphi_{b}} d \varphi \frac{p^{2}}{(k p)}
\end{aligned}
$$$$
\times\left(\frac{d\left(\Gamma_{\alpha} t_{\beta}^{\alpha} \Gamma_{\beta}\right)}{d \varphi}\left(\Gamma_{\alpha} t_{\beta}^{\alpha} \Gamma_{\beta}\right)\right)^{2}+\frac{i g}{16}
$$$$
\times \int_{\varphi_{a}}^{\varphi_{b}} d \varphi \frac{p^{2}}{(k p)}
$$$$
\times\left(\frac{d\left(\Gamma_{\alpha} t_{\beta}^{\alpha} \Gamma_{\beta}\right)}{d \varphi}\left(\Gamma_{\alpha} t_{\beta}^{\alpha} \Gamma_{\beta}\right)\right) \chi
$$$$
+\frac{g}{64} \times \int_{\varphi_{a}}^{\varphi_{b}} d \varphi \frac{1}{(k p)}
$$ 


$$
\begin{gathered}
\left.\times\left(\frac{d\left(\Gamma_{\alpha} t_{\beta}^{\alpha} \Gamma_{\beta}\right)}{d \varphi}\left(\Gamma_{\alpha} t_{\beta}^{\alpha} \Gamma_{\beta}\right)\right)^{2} \chi\right) \\
+\frac{g^{2}}{64} \int_{\varphi_{1 a}}^{\varphi_{1 b}} d \varphi_{1} \\
\left.\times \int_{\varphi_{2 a}}^{\varphi_{2 b}} d \varphi_{2} \frac{1}{(k p)}\left(b \varphi_{1}+c \varphi_{2}\right)(k \theta)\left(\Gamma_{\alpha} t_{\beta}^{\alpha} \Gamma_{\beta}\right)\right]\left.\right|_{\theta=9=0} .
\end{gathered}
$$

The variable $\chi[16,19]$ can be eliminated by integration, and we obtain

$$
\begin{aligned}
& \widetilde{S}\left(x_{b}, x_{a}\right) \exp \left(i \gamma \frac{\delta_{L}}{\delta \theta}\right) \int_{0}^{\infty} d \lambda \\
& \times \int \frac{d^{4} p}{(2 \pi)^{4}} \exp \left\{i p\left(x_{b}-x_{a}\right)+i \lambda\left(p^{2}-M^{2}\right)\right\} \\
& \times\left(\frac{1}{2}\left((p \theta)+M \theta^{5}\right)\right) \\
& \times \exp \left[-\frac{g}{16}\left((k \theta)+i \frac{(k \theta)}{(k p)^{2}} p^{2}+\frac{g}{8}(k \theta)\right)\right. \\
& \quad \times \int_{\varphi_{a}}^{\varphi_{b}} d \varphi\left(\frac{d\left(\Gamma_{\alpha} t_{\beta}^{\alpha} \Gamma_{\beta}\right)}{d \varphi}\left(\Gamma_{\alpha} t_{\beta}^{\alpha} \Gamma_{\beta}\right)\right) \\
& \quad+\frac{1}{32} \frac{(k \theta)}{(k p)} \int_{\varphi_{a}}^{\varphi_{b}} d \varphi\left(\frac{d\left(\Gamma_{\alpha} t_{\beta}^{\alpha} \Gamma_{\beta}\right)}{d \varphi}\left(\Gamma_{\alpha} t_{\beta}^{\alpha} \Gamma_{\beta}\right)\right) \\
& \quad \times(k \cdot 9)+\frac{(k \theta)}{(k p)}\left(-\frac{g}{64(k p)}+\frac{(k p)}{128}\right) \\
&\left.\times \int_{\varphi_{2 a}}^{\varphi_{2 b}} d \varphi_{2}\left(b \varphi_{1}+c \varphi_{2}\right)\left(\Gamma_{\alpha} t_{\beta}^{\alpha} \Gamma_{\beta}\right)\right]\left.\right|_{\theta=9=0} ^{\varphi_{1 b}} d \varphi\left(\frac{d\left(\Gamma_{\alpha} t_{\beta}^{\alpha} \Gamma_{\beta}\right)}{d \varphi}\left(\Gamma_{\alpha} t_{\beta}^{\alpha} \Gamma_{\beta}\right)\right)+\frac{g^{2}}{64} \frac{(k \theta)}{(k p)}
\end{aligned}
$$

where the calculations have been simplified thanks to proprieties of field.
The matrices $\gamma^{\mu}$ are reintroduced by using the following relations:

$$
\begin{gathered}
\left.\exp \left(i\left(\gamma \frac{\delta_{L}}{\delta \theta}\right)\right) f(\theta)\right|_{\theta=0} \\
=\left.f\left(\frac{\partial_{L}}{\partial \theta}\right) \exp (i(\gamma \theta))\right|_{\theta=0}, \\
\frac{\partial_{L}^{2}}{\partial \theta^{\alpha} \partial \theta^{\beta}} \theta_{\mu} \theta_{\nu} \gamma^{\mu} \gamma^{\nu}=-2 i \sigma_{\alpha \beta}, \\
\exp (i(\gamma \theta))=1+i(\gamma \theta) \\
-\frac{1}{2} \theta_{\mu} \theta_{\nu} \gamma^{\mu} \gamma^{\nu} \\
+\frac{i}{6} \theta_{\mu} \theta_{\nu} \theta_{\sigma} \gamma^{\mu} \gamma^{\nu} \gamma^{\sigma} \\
+\theta_{0} \theta_{1} \theta_{2} \theta_{3} \gamma^{5},
\end{gathered}
$$

where

$$
\begin{aligned}
f(\theta) & \frac{1}{2}\left((p \theta)+M \theta^{5}\right) \\
\times \exp \{- & \frac{g}{16}\left((k \theta)+i \frac{(k \theta)}{(k p)^{2}} p^{2}+\frac{g}{8}(k \theta)\right) \\
& \times \int_{\varphi_{a}}^{\varphi_{b}} d \varphi\left(\frac{d\left(\Gamma_{\alpha} t_{\beta}^{\alpha} \Gamma_{\beta}\right)}{d \varphi}\left(\Gamma_{\alpha} t_{\beta}^{\alpha} \Gamma_{\beta}\right)\right) \\
& +\frac{1}{32} \frac{(k \theta)}{(k p)} \\
& \times \int_{\varphi_{a}}^{\varphi_{b}} d \varphi\left(\frac{d\left(\Gamma_{\alpha} t_{\beta}^{\alpha} \Gamma_{\beta}\right)}{d \varphi}\left(\Gamma_{\alpha} t_{\beta}^{\alpha} \Gamma_{\beta}\right)\right) \\
& \times(k \cdot \vartheta)+\frac{(k \theta)}{(k p)}\left(-\frac{g}{64(k p)}+\frac{(k p)}{128}\right) \\
& \times \int_{\varphi_{a}}^{\varphi_{b}} d \varphi\left(\frac{d\left(\Gamma_{\alpha} t_{\beta}^{\alpha} \Gamma_{\beta}\right)}{d \varphi}\left(\Gamma_{\alpha} t_{\beta}^{\alpha} \Gamma_{\beta}\right)\right)^{2} \\
& +\frac{g^{2}}{64} \frac{(k \theta)}{(k p)} \int_{\varphi_{1 a}}^{\varphi_{1 b}} d \varphi_{1} \\
& \left.\times \int_{\varphi_{2 a}}^{\varphi_{2 b}} d \varphi_{2}\left(b \varphi_{1}+c \varphi_{2}\right)\left(\Gamma_{\alpha} t_{\beta}^{\alpha} \Gamma_{\beta}\right)\right\}
\end{aligned}
$$


Then

$$
\begin{aligned}
& \tilde{S}\left(x_{b}, x_{a}\right) \\
& =\int_{0}^{\infty} d \lambda \\
& \quad \times \int \frac{d^{4} p}{(2 \pi)^{4}} \exp \left\{i p\left(x_{b}-x_{a}\right)+i \lambda\left(p^{2}-M^{2}\right)\right\} \\
& \quad \times \exp \left\{\frac{i}{(k p)}\right. \\
& \quad \times\left(\frac{g}{64} \frac{(p \sigma k)}{(k p)}\right. \\
& \left.\left.\quad \times \int_{k x_{a}}^{k x_{b}} d \varphi\left(b \frac{k_{1}}{k}+c \frac{k_{2}}{k}\right) \varphi \frac{d m(\varphi)}{d \varphi}\right)\right\},
\end{aligned}
$$

and after having eliminated $\lambda$ by integration, we have

$$
\begin{aligned}
& \tilde{S}\left(x_{b}, x_{a}\right) \\
& =\frac{1}{(2 \pi)^{4}} \\
& \times \int \frac{d^{4} p(-(\gamma p)+M)}{p^{2}-M^{2}+i O} \exp \left\{i p\left(x_{b}-x_{a}\right)\right\} \\
& \times \exp \left\{\frac{i}{(k p)}\right. \\
& \quad \times\left(\frac{g}{64} \frac{(p \sigma k)}{(k p)}\right. \\
& \left.\left.\quad \times \int_{k x_{a}}^{k x_{b}} d \varphi\left(b \frac{k_{1}}{k}+c \frac{k_{2}}{k}\right) \varphi \frac{d m(\varphi)}{d \varphi}\right)\right\},
\end{aligned}
$$

and an integration by part of the exponent of exponential $\widetilde{S}\left(x_{b}, x_{a}\right)$ has the following form:

$$
\begin{aligned}
& \widetilde{S}\left(x_{b}, x_{a}\right) \\
& =\frac{1}{(2 \pi)^{4}} \int \frac{d^{4} p(-(\gamma p)+M)}{p^{2}-M^{2}+i 0^{+}} \exp \left\{i p\left(x_{b}-x_{a}\right)\right\} \\
& \quad \times \exp \left\{\frac{i g}{64(k p)^{2}}(p \sigma k)\right.
\end{aligned}
$$

$$
\begin{gathered}
\times\left[\left(\left(\frac{k_{1}}{k} b\left(\varphi_{b}\right)+\frac{k_{2}}{k} c\left(\varphi_{b}\right)\right) \varphi_{b} m\left(\varphi_{b}\right)\right.\right. \\
\left.\quad-\left(\frac{k_{1}}{k} b\left(\varphi_{a}\right)+\frac{k_{2}}{k} c\left(\varphi_{a}\right)\right) \varphi_{a} m\left(\varphi_{a}\right)\right) \\
\quad-\int_{k x_{a}}^{k x_{b}} d \varphi \\
\times\left(\frac{k_{1}}{k}\left(b(\varphi)+\varphi b^{\prime}(\varphi)\right)\right. \\
\left.\left.\left.\quad+\frac{k_{2}}{k}\left(c(\varphi)+\varphi c^{\prime}(\varphi)\right)\right) m(\varphi)\right]\right\},
\end{gathered}
$$

and after some manipulations, the Green function for the Dirac particles moving in the non-Abelian field in the local approach is finally

$$
\begin{aligned}
& \widetilde{S}\left(x_{b}, x_{a}\right) \\
& =\int \frac{d^{4} p}{(2 \pi)^{4}} \frac{1}{p^{2}-M^{2}+i 0^{+}} \exp \left\{i p\left(x_{b}-x_{a}\right)\right\} \\
& \times \frac{g}{64} \frac{1}{(k p)^{2}} \\
& \times\left[\left(1+(p \sigma k)\left(\frac{k_{1}}{k} b\left(\varphi_{b}\right)+\frac{k_{2}}{k} c\left(\varphi_{b}\right)\right) \varphi_{b} m\left(\varphi_{b}\right)\right)\right. \\
& \times(-(\gamma p)+M) \\
& \left.\times\left(1-(p \sigma k)\left(\frac{k_{1}}{k} b\left(\varphi_{a}\right)+\frac{k_{2}}{k} c\left(\varphi_{a}\right)\right) \varphi_{a} m\left(\varphi_{a}\right)\right)\right] \\
& \times \exp \left\{i \left[p\left(x_{b}-x_{a}\right)-\frac{g(p \sigma k)}{64(k p)^{2}}\right.\right. \\
& \times\left(\int_{k x_{a}}^{k x_{b}} d \varphi\right. \\
& \times\left[\frac{k_{1}}{k}\left(b(\varphi)+\varphi b^{\prime}(\varphi)\right)+\frac{k_{2}}{k}\right. \\
& \left.\left.\left.\left.\times\left(c(\varphi)+\varphi c^{\prime}(\varphi)\right)\right] m(\varphi)\right)\right]\right\},
\end{aligned}
$$

which is obviously symmetrical with respect to $x_{a}$ and $x_{b}$. From the residues related to two poles

$$
p_{ \pm}^{0}= \pm \omega-i 0^{+}= \pm\left(\mathbf{p}^{2}+M^{2}\right)^{-1 / 2}-i 0^{+}
$$


and after having introduced the projectors on states of positive and negative energy [20],

$$
\begin{gathered}
\Lambda_{+}=\sum_{ \pm s} u(p, s) \bar{u}(p, s)=\frac{\gamma p+M}{2 M}, \\
\Lambda_{-}=\sum_{ \pm s} v(p, s) \bar{v}(p, s)=\frac{-\gamma p+M}{2 M}
\end{gathered}
$$

we obtain the following spectral decomposition:

$$
\begin{aligned}
S\left(x_{b}, x_{a}\right)= & -i \theta\left(t_{b}-t_{a}\right) \\
& \times \int d^{3} p \sum_{ \pm s} \Psi_{s, p}^{+}\left(x_{b}\right) \bar{\Psi}_{s, p}^{+}\left(x_{a}\right) \\
& +i \theta\left(t_{b}-t_{a}\right) \int d^{3} p \\
& \times \sum_{ \pm s} \Psi_{s, p}^{-}\left(x_{b}\right) \overline{\Psi_{s, p}}\left(x_{a}\right)
\end{aligned}
$$

where finally the wave functions related to particle and to antiparticle are, respectively,

$$
\begin{aligned}
& \Psi_{s, p}^{+}(x) \\
& =\frac{1}{(2 \pi)^{3 / 2}}\left(\frac{M}{p^{0}}\right)^{1 / 2} \\
& \times(1+(p \sigma k) \\
& \left.\times\left(\frac{k_{1}}{k} b(k x)+\frac{k_{2}}{k} c(k x)\right) \varphi_{b} m(k x)\right) u(p, s) \\
& \times \exp \left\{i \left[p x-\frac{g(p \sigma k)}{64(k p)^{2}}\right.\right. \\
& \times\left[\int^{k x} d \varphi\right. \\
& \times\left(\frac{k_{1}}{k}\left(b(\varphi)+\varphi b^{\prime}(\varphi)\right)\right. \\
& \left.+\frac{k_{2}}{k}\left(c(\varphi)+\varphi c^{\prime}(\varphi)\right)\right) \\
& \times m(\varphi)]],
\end{aligned}
$$

$$
\begin{aligned}
& \Psi_{s, p}^{-}(x) \\
& =\frac{1}{(2 \pi)^{3 / 2}}\left(\frac{M}{p^{0}}\right)^{1 / 2} \\
& \quad \times(1-(p \sigma k) \\
& \quad \times\left(\frac{k_{1}}{k} b(k x)+\frac{k_{2}}{k} c(k x)\right) \\
& \left.\quad \times \varphi_{a} m(k x)\right) v(p, s)
\end{aligned}
$$

$$
\begin{aligned}
\times \exp \{-i[p x & +\frac{g(p \sigma k)}{64(k \cdot p)^{2}} \\
\times & {\left[\int^{k x} d \varphi\right.} \\
& \times\left(\frac{k_{1}}{k}\left(b(\varphi)+\varphi b^{\prime}(\varphi)\right)\right. \\
& \left.\left.\left.\left.+\frac{k_{2}}{k}\left(c(\varphi)+\varphi c^{\prime}(\varphi)\right)\right) m(\varphi)\right]\right]\right\} .
\end{aligned}
$$

$u(p, s)$ and $v(p, s)$ are the spinors solutions of the free Dirac equation and are

$$
\begin{aligned}
& \bar{u}(p, s) u(p, s)=1 \\
& \bar{v}(p, s) v(p, s)=-1 .
\end{aligned}
$$

\section{Conclusion}

In the present paper, the Green function related to Dirac particle moving in a non-abelian field has been determined in the local approach of path integral. The obtained wave functions from the spectral decomposition are in accordance with those obtained by direct resolution of Dirac equation [1].

Using only shifts related to properties of field, the integrations have been easily performed, and it is remarkable to notice that the selected equations by arguments of $\delta$ functions which are appeared during calculation can be also obtained from the classical mechanics (cf. Appendix).

\section{Appendix}

In local approach, the action is

$$
\begin{aligned}
A=\int_{0}^{\lambda} d \tau & \\
& \times\left[p \dot{x}+p^{2}-M^{2}-\frac{i}{16}\right. \\
& \times(p \Psi)(k \Psi)\left(t_{\beta}^{\alpha} \Upsilon_{\alpha}^{\prime} \Upsilon_{\beta}\right) \\
& -i \frac{g}{4}(p \Psi)(k \Psi)
\end{aligned}
$$




$$
\begin{aligned}
& \times\left(b\left(k_{1} x\right)+c\left(k_{2} x\right)\right)\left(t_{\beta}^{\alpha} \Upsilon_{\alpha} \Upsilon_{\beta}\right) \\
& +\chi\left(-(p \Psi)+\frac{i}{32}(k \Psi)\left(\Psi\left(t_{\beta}^{\alpha} \Upsilon_{\alpha}^{\prime} \Upsilon_{\beta}\right)\right)\right. \\
& +i \frac{g}{8}(k \Psi)\left(b\left(k_{1} x\right)+c\left(k_{2} x\right)\right) \\
& \left.\left.\times\left(t_{\beta}^{\alpha} \Upsilon_{\alpha} \Upsilon_{\beta}\right)-M \Psi^{5}\right)-i \Psi \dot{\Psi}-i \Upsilon \dot{\Upsilon}\right]
\end{aligned}
$$

and the equations of movement are, respectively,

$$
\begin{aligned}
& \frac{\delta A}{\delta x^{\mu}(s)}=0: \dot{p}=-(k \Psi) \\
& \times\left\{-i \frac{g}{4}(k \cdot \Psi)\right. \\
& \times\left[\left(k_{1} b^{\prime}\left(k_{1} x\right)+k_{2} c^{\prime}\left(k_{2} x\right)\right)\right. \\
& \times\left(t_{\beta}^{\alpha} \Upsilon_{\alpha} \Upsilon_{\beta}\right) \\
& +\left(b\left(k_{1} x\right)+c\left(k_{2} x\right)\right) \\
& \left.\times\left(t_{\beta}^{\alpha} k \Upsilon_{\alpha}^{\prime} \Upsilon_{\beta}+t_{\beta}^{\alpha} \Upsilon_{\alpha} k \Upsilon_{\beta}^{\prime}\right)\right] \\
& +\chi\left(\frac{i}{32}\left((k \Psi)\left(t_{\beta}^{\alpha} k \Upsilon_{\alpha}^{\prime \prime} \Upsilon_{\beta}+t_{\beta}^{\alpha} \Upsilon_{\alpha}^{\prime} k \Upsilon_{\beta}^{\prime}\right)\right)+i \frac{g}{8} k\right. \\
& \times\left[\left(k_{1} b^{\prime}\left(k_{1} x\right)+k_{2} c^{\prime}\left(k_{2} x\right)\right)\right. \\
& \times\left(t_{\beta}^{\alpha} \Upsilon_{\alpha} \Upsilon_{\beta}\right) \\
& +\left(k_{1} b\left(k_{1} x\right)+k_{2} c\left(k_{2} x\right)\right) \\
& \left.\left.\left.\times\left(t_{\beta}^{\alpha} k \Upsilon_{\alpha}^{\prime} \Upsilon_{\beta}+t_{\beta}^{\alpha} \Upsilon_{\alpha} k \Upsilon_{\beta}^{\prime}\right)\right]\right)\right\}, \\
& \frac{\delta A}{\delta p^{\mu}(s)}=0: \dot{x}=-2 p \\
& +i \frac{g}{4} \Psi(k \Psi) \\
& \times\left(b\left(k_{1} x\right)+c\left(k_{2} x\right)\right)\left(t_{\beta}^{\alpha} \Upsilon_{\alpha}^{\prime} \Upsilon_{\beta}\right) . \\
& \frac{\delta A}{\delta \Psi^{\mu}}=0: \dot{\Psi}=\frac{1}{16} \Psi(k \Psi) \\
& \times\left(t_{\beta}^{\alpha} \Upsilon_{\alpha}^{\prime} \Upsilon_{\beta}\right)+\frac{g}{8} \Psi(k \Psi)
\end{aligned}
$$

$$
\begin{aligned}
& \times\left(b\left(k_{1} x\right)+c\left(k_{2} x\right)\right)\left(t_{\beta}^{\alpha} \Upsilon_{\alpha} \Upsilon_{\beta}\right), \\
\dot{\Psi}^{5} & =i M \chi: \Psi^{5}=i M \tau \chi+\text { const., } \\
\frac{\delta A}{\delta \Upsilon^{\mu}}= & 0: \dot{\Upsilon}=\frac{1}{16}(p \Psi)(k \Psi) \\
& \times\left(t_{\beta}^{\alpha} \Upsilon_{\alpha}^{\prime} \Upsilon_{\beta}\right)+\frac{g}{4}(p \Psi)(k \Psi) \\
& \times\left(b\left(k_{1} x\right)+c\left(k_{2} x\right)\right)\left(t_{\beta}^{\alpha} \Upsilon_{\alpha} \Upsilon_{\beta}\right) .
\end{aligned}
$$

After multiplication by $k_{\mu}$ and $k_{i}(i=1,2)$, we obtain the following relations:

$$
\begin{gathered}
k \dot{p}=0, \quad k p=\text { const. } \\
\dot{\varphi}=k \dot{x}=-2 k p, \\
\dot{\varphi}_{i}=k_{i} \dot{x}=-2 k_{i} p, \\
k \dot{\Psi}=0 .
\end{gathered}
$$

\section{References}

[1] A. Obukhov, V. K. Perez-Fernandez, and V. R. Khalilov, "Exact solutions of the equations of motions of quarks in a non-abelian field of a flat color wave," Russian Physics Journal, vol. 29, no. 12, pp. 1016-1018, 1986.

[2] S. Coleman, "Non-Abelian plane waves," Physics Letters B, vol. 70, no. 1, pp. 59-60, 1977.

[3] D. M. Gitman and M. Sh. Shvartsman, "Spinor and isospinor structure of relativistic particle propagators," Physics Letters B, vol. 318, no. 3-4, 122 pages, 1993.

[4] J. Schwinger, "On gauge invariance and vacuum polarization," Physical Review, vol. 82, pp. 664-679, 1951.

[5] E. S. Fradkin, "Application of functional methods in quantum field theory and quantum statistics. II," Nuclear Physics B, vol. 76, pp. 588-624, 1966.

[6] B. M. Barbashov, "Functional integrals in quantum electrodynamics and the infrared limit of the Green's functions," JETP, vol. 21, pp. 402-410, 1965.

[7] A. I. Nikishov and V. I. Ritus, "Radiation spectrum of an electron moving in a constant electric field," Zhurnal Éksperimental noi i Teoreticheskoi Fiziki, vol. 56, no. 6, p. 2035, 1969.

[8] A. Barducci, F. Bordi, and R. Casalbuoni, "Path integral quantization of spinning particles interacting with crossed external electromagnetic fields," Nuovo Cimento B, vol. 64, no. 2, pp. $287-$ 315, 1981.

[9] A. Batalin and E. S. Fradkin, "Quantum electrodynamics in external fields. I," Theoretical and Mathematical Physics, vol. 5, no. 2, pp. 1080-1100, 1970.

[10] S. P. Gavrilov, D. M. Gitman, and Sh. M. Shvartsman, "Green's functions in an external electric field," Soviet Journal of Nuclear Physics, vol. 29, pp. 1392-1405, 1979.

[11] S. Zeggari, T. Boudjedaa, and L. Chetouani, "Path integral for dirac particle in plane wave field," Physica Scripta, vol. 64, no. 4, p. 285, 2001. 
[12] N. Boudiaf, T. Boudjedaa, and L. Chetouani, "Path integral for spinning particle in the plane wave field: global and local projections," The European Physical Journal C, vol. 20, no. 3, pp. 585-591, 2001.

[13] N. Boudiaf, A. Merdaci, and L. Chetouani, "Path integral solution for dirac particle in a constant electric field," Journal of Physics A, vol. 42, no. 1, p. 12, 2009.

[14] S. Haouat and L. Chetouani, "Worldline path integrals for a dirac particle in a weak gravitational plane wave," The European Physical Journal C, vol. 53, no. 2, pp. 289-294, 2008.

[15] E. S. Fradkin, D. M. Gitman, and M. Sh. Shvartsman, Quantum Electro-Dynamics: With Unstable Vacuum, Springer, Berlin, Germany, 1991.

[16] D. M. Gitman and A. V. Saa, "Quantization of a spinning particle with anomalous magnetic moment," Classical and Quantum Gravity, vol. 10, no. 8, pp. 1447-1460, 1993.

[17] E. S. Fradkin and D. M. Gitman, "Path-integral representation for the relativistic particle propagators and BFV quantization," Physical Review D, vol. 44, no. 10, pp. 3230-3236, 1991.

[18] D. M. Gitman, "Path integrals and pseudoclassical description for spinning particles in arbitrary dimensions," Nuclear Physics B, vol. 488, no. 1-2, pp. 490-512, 1997.

[19] C. Alexandrou, R. Rosenfelder, and A. W. Schreiber, "Worldline path integral for the massive Dirac propagator: a fourdimensional approach," Physical Review A, vol. 59, no. 3, pp. 1762-1776, 1999.

[20] J. D. Bjorken and S. D. Drell, Relativistic Quantum Mechanics, McGraw-Hill, New York, NY, USA, 1964. 

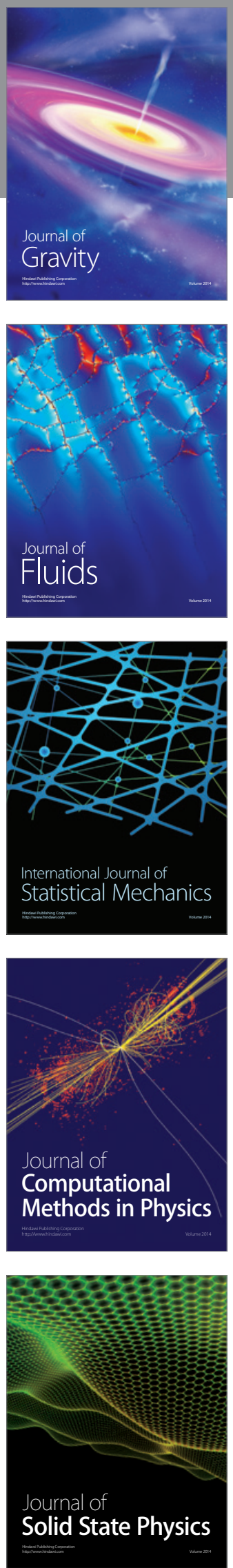

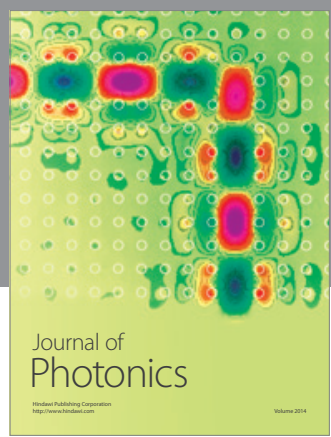

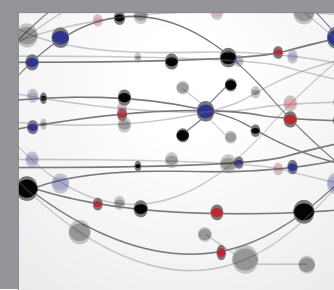

The Scientific World Journal

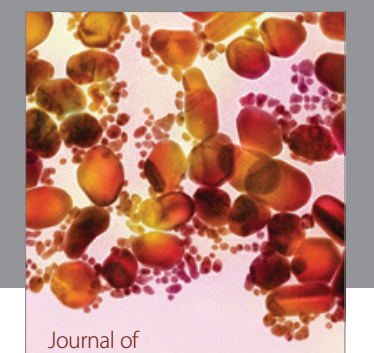

Soft Matter
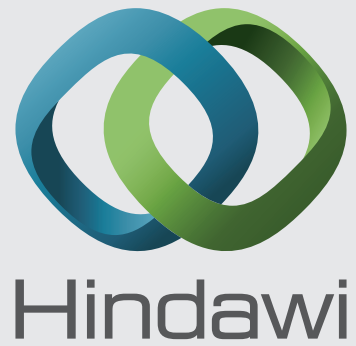

Submit your manuscripts at

http://www.hindawi.com
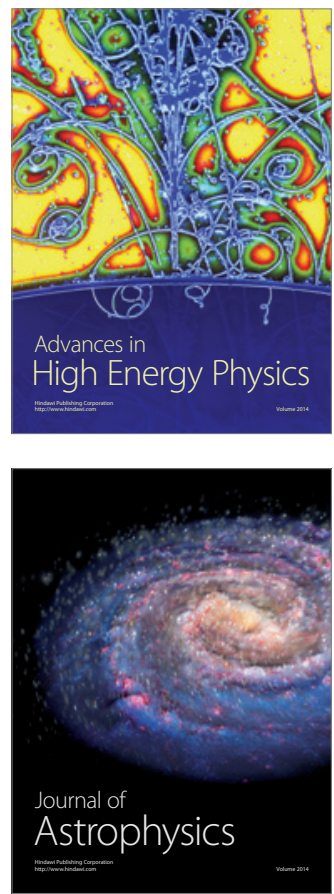
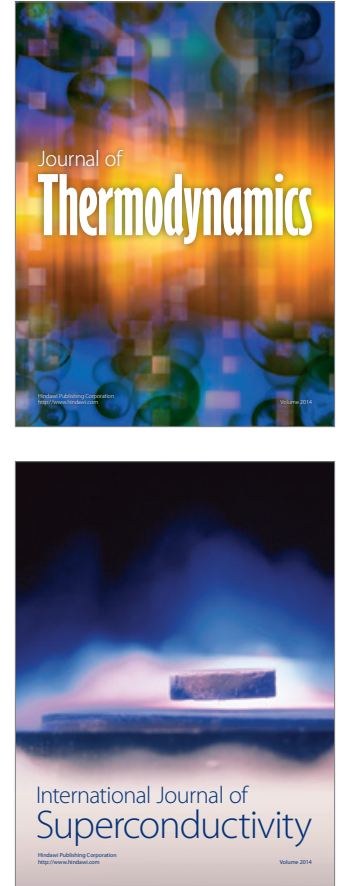
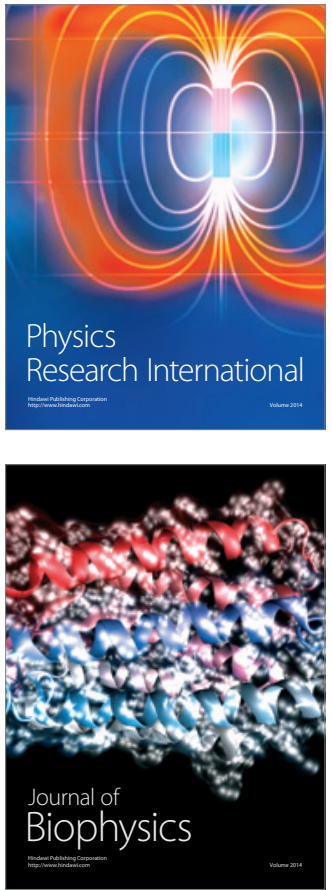
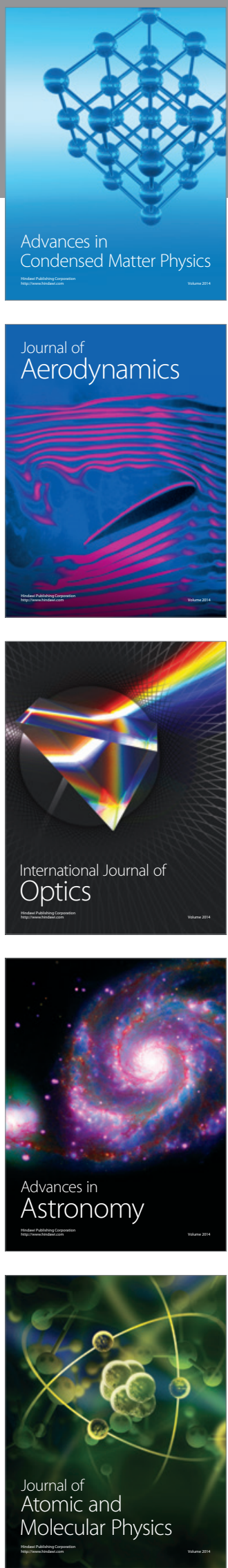